\title{
Facilitating Peer Support for Recovery from Substance Use Disorders
}

Sabirat Rubya

University of Minnesota

Minneapolis, MN 55455, USA

rubya001@umn.edu
Permission to make digital or hard copies of part or all of this work for personal or classroom use is granted without fee provided that copies are not made or distributed for profit or commercial advantage and that copies bear this notice and the full citation on the first page. Copyrights for third-party components of this work must be honored. For all other

uses, contact the Owner/Author.
Copyright is held by the owner/author(s)

CHI'17 Extended Abstracts, May 06-11, 2017, Denver, CO, USA ACM $978-1-4503-4656-6 / 17 / 05$.

http://dx.doi.org/10.1145/3027063.3048431

\begin{abstract}
Twelve-step fellowships is an effective maintenance program for millions of people trying to recover from substance use disorders. Attending face-to-face and online twelve-step meetings is considered to play a vital role for exchanging support and for encouraging continued abstinence from addiction. Currently, there are very few technologies that effectively support this group in exchanging peer support through synchronous communication. I conducted a questionnaire and an interview study to better understand the challenges and needs of this group. This work discusses some design opportunities and ongoing work to facilitate peer support for recovery from substance use disorders.
\end{abstract}

\section{Author Keywords}

Peer support; twelve-step meetings; online recovery communities; computer-mediated communication.

\section{ACM Classification Keywords}

H.5.2. [Information Interfaces and Presentation]: User Interfaces: User-Centered Design.

\section{Introduction}

Substance use disorders are prevalent and high-impact health conditions among US population. It is estimated to cost the United States $\$ 374$ billion per year, with $11.9 \%$ of Americans developing a dependence on the 
substance [1]. Treatments for recovery from these substance use disorders are effective when clinical intervention is coupled with long-term maintenance programs. One of the most effective and popular maintenance program is twelve-step fellowships (e.g., AA and NA). AA and NA focus on providing face-to-face support through sharing problems and experiences in meetings. Recently this approach has been expanded to online communities, too. Previous studies show that face-to-face and online meeting attendance is effective for long-term abstinence $[7,10]$. Despite the abundance of technology to support in-person connections, there has been very few technologies to help the peersupport process in these special groups. This work attempts to expand our understanding of the needs of these meeting attendees in order to inform the design of technologies to address these challenges and describes some of the technologies we have started to develop informed by the findings from a questionnaire and an interview study.

I begin with an overview of related literature on effectiveness of peer support in different online health communities. I then give an overview of the methods with demographics of the participants. Finally, I present the major themes that resulted from the semistructured interviews of 14 members of the largest online recovery community (www.intherooms.com or ITR) and discuss some design opportunities to help them exchange support through synchronous communication.

\section{Related Work}

Recent work in HCI and CSCW has begun to focus on how technology can help facilitate social support in online health communities. I build on this significant body of work and give an overview of other technologies to maintain abstinence from addiction.

Peer Support in Online Health Communities

There have been many investigations of social support in online communities focused on different health conditions such as weight loss [5], cancer [3], addiction [7], and infertility [9]. Such communities provide informational and emotional support, encouragement, and friendship that are commonly thought to reduce negative health outcomes as well as encourage active community engagement $[2,8]$. Several of this diverse body of work have investigated effectiveness of synchronous peer support in recovery from substance use disorder particularly by attending twelve-step meetings $[7,10]$.

The findings of these studies were helpful in informing my work. However, in this work, I particularly explore the challenges in the peer support process faced by people trying to recover from substance use disorder.

Role of Technology in Recovery

Several investigations provide some implications for designing forums for sustainable recovery and for illuminating poorly understood aspects of addiction $[6,10]$. Another body of work has designed and developed mobile applications for recovering addicts. For example, SoberDiary incorporated an app with a breathalyzer to log drinking behavior and suggest skills to reduce alcohol cravings [3]. A-CHESS [5] users work with a clinical support team, share experiences in a discussion board, and track proximity to problematic people or locations. While these investigations highlight the potential for technology to help people recover in a clinical program, this work focuses on understanding 


\begin{tabular}{l|r}
$\begin{array}{l}\text { Source of getting } \\
\text { meeting schedule }\end{array}$ & $\begin{array}{r}\text { \# of } \\
\text { Parti- } \\
\text { cipants }\end{array}$ \\
\hline Google & 6 \\
Booklet/pamphlet & 2 \\
Local AA & \\
Websites & 3 \\
Calling hotline/ & \\
intergroup & 1 \\
Sponsor & 1 \\
ITR List & 3 \\
Email & 1
\end{tabular}

Table 1: Sources to know about face-to-face meetings

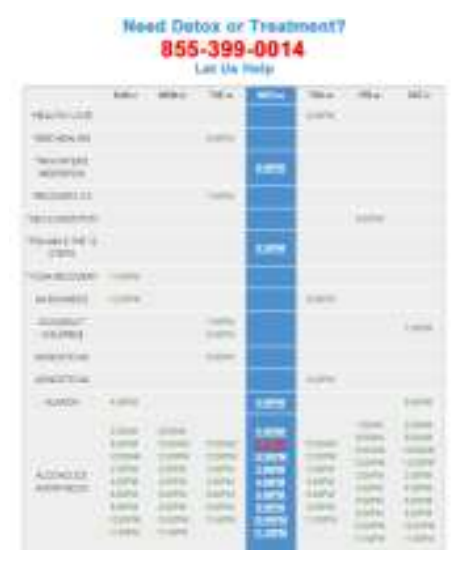

Figure 1: List of Online Video Meetings on ITR.com the potential role of technology in peer support as part of a long-term maintenance program of relapse prevention.

\section{Methods}

I conducted a questionnaire and an interview study. A link to a questionnaire was distributed as a paid banner advertisement on the homepage of ITR for one month, as well as advertised in the weekly newsletter. 285 participants completed the questionnaire. The average age of these participants was 49 years $(S D=11.5)$ with $61 \%$ of them being female. Participants' geographic location covered 16 different countries, with majority of them $(72 \%)$ being from US. The demographic information of the participants was representative of active users of the community [7]. Survey participants had an average of 8.5 years of recovery time $(S D=9.5$ years).

Participation in different twelve-step meetings was selfreported as an important part of recovery and in order to know more about experience of these meeting attendance I recruited $14 \mathrm{AA} / \mathrm{NA}$ members from the same community where the questionnaire was deployed. The participants had an average of 8.6 years of recovery time ( $S D=9.75$ years). They represented four different countries and nine of them were female. These participants had frequent face-to-face or online meeting attendances or both in the past three months. I conducted a 90-minute semi-structured interview with each of them. The questions asked focus on use of technology in finding or attending meetings, difference between their experiences in attending face-to-face and online meetings, reasons of attending (or not attending) face-to-face meetings, and challenges or problems faced in finding or attending meetings.
The transcripts were analyzed using a data-driven approach. Statements of interest (open codes) were extracted and grouped by different themes. The open codes were rearranged until a set of distinct themes emerged. I present an overview of the results in thematic clusters in the next section.

\section{Results}

The questionnaire responses revealed that regular meeting attendees tend to have greater commitment to their recovery with higher average of years in recovery. This supports the positive impacts of meeting attendance in recovery in previous studies. The interview data gives us more insight on challenges people face in finding or attending these meetings.

\section{Questionnaire}

$67 \%$ of the survey responders reported to attend $2-3$ meetings per week. The questionnaire also asked the participants to self-evaluate their commitment to recovery on the basis of a modified 7-item AAI (Alcoholics Anonymous Involvement) scale [4]. The self-reported average involvement score was 32.5 $(S D=4.3)$ out of 40 for those who attend at least one meeting per week vs $30.2(S D=5.6)$ for those who do not attend meetings (higher score means greater involvement). The average recovery time was also higher $(M=9.5, S D=10.3)$ for participants who reported to attend online, face-to-face, or both types of meetings.

\section{Interviews}

IMPORTANCE OF MEETING ATTENDANCE

Previous research on recovery has emphasized the importance of attending twelve-step meetings for abstinence [10]. The questionnaire and the interview 
P6: "I went to visit family and I looked up for some types of face-to-face meeting, and one of the meetings that I was going to go to, I drove down the road wasn't there. I really really needed a meeting, I didn't have my computer. And it was awful."

P8: "If there is a small group of people that they are comfortable with each other then it tend to be more open in a face-to-face meeting than it could be possibly online."

P4: "It feels good if you know the people in the meetings. The message and the peoplemake a meeting effective"

P7: "I think, if the technology was available back then, I would have turned to some online meetings; cause I would have felt some more acceptance. That people wouldn't have been so judgmental of me"

Figure 2: Quotes from the interviews Revealing Challenges in Finding or Attending Meetings studies revealed this point, with many participants mentioning the meetings as sources of encouragement and support in their recovery processes.

Many of the participants, especially newcomers, consider meetings as a way of avoiding self-destructive and delusional thinking, loneliness, and isolation. Many newcomers see these meetings as a place to hear about "experience, strength, and hope" from others, whereas oldtimers (members who have fairly longer recovery time) view it as a way to "pass on the love that's once being given" and to "let people know that somebody cares". Some participants considered it as a part of their spiritual contentment. Meeting different people in the meetings enable them to share problems with like-minded others, as well as to know about diverse perspectives.

Face-to-face meetings typically have a consistent set of attendees which makes it easy to identify a newcomer. Newcomers are usually nervous; hence a welcoming gesture enables them to overcome their struggle. Besides, they can form close friendship with people from their local meetings and even find a sponsor.

FACE-TO-FACE AND ONLINE MeETings

Ten out of the fourteen participants attended both faceto-face and online meetings. Although online meetings have been receiving attention recently, most of the participants thought that the online meetings were useful only if they were attended as a supplement of the face-to-face meetings, not as a replacement.

Challenges in Finding the Right Meeting Despite the popularity of the twelve-step programs and potential of meeting attendance for positive impact, there is no global list of AA meetings. The fourteen interview participants mentioned seven different sources for finding right meetings for themselves (Table 1). Meeting attendees usually know about their groups' meetings but lack of a global meeting list makes it hard to find the right meeting when they are traveling. Different websites of different AA regional intergroups contain meeting lists in different formats (e.g., webpage, PDF, scanned images, etc) and accessing these lists from different devices become more difficult. This is also a core problem for newcomers who have a chance of losing faith in the program if they feel the immediacy of attending a meeting but cannot find one.

Types of meetings members want to join depends on different factors: fellowship, recovery days, intention of attending a meeting, etc. People with more recovery days are usually willing to find a lot of newcomers so that they can pass on their knowledge, whereas members who are nervous or shy prefer to attend meetings where they have familiar people (e.g., people from their face-to-face meetings). ITR provides a list of online meetings throughout the week (Figure 1) but there is no available list of probable meeting participants. Figure 2 shows some relevant quotes from our interview participants illustrating these problems.

\section{Design Instantiations}

Global List of AA Meetings

Since AA meeting lists are catalogues at regional levels (e.g., towns, counties), meeting attendees find it difficult to search for nearby meetings or stay connected while traveling outside of their usual area.

I have started to build a system using machine learning and crowdsourcing approaches to create a global AA 


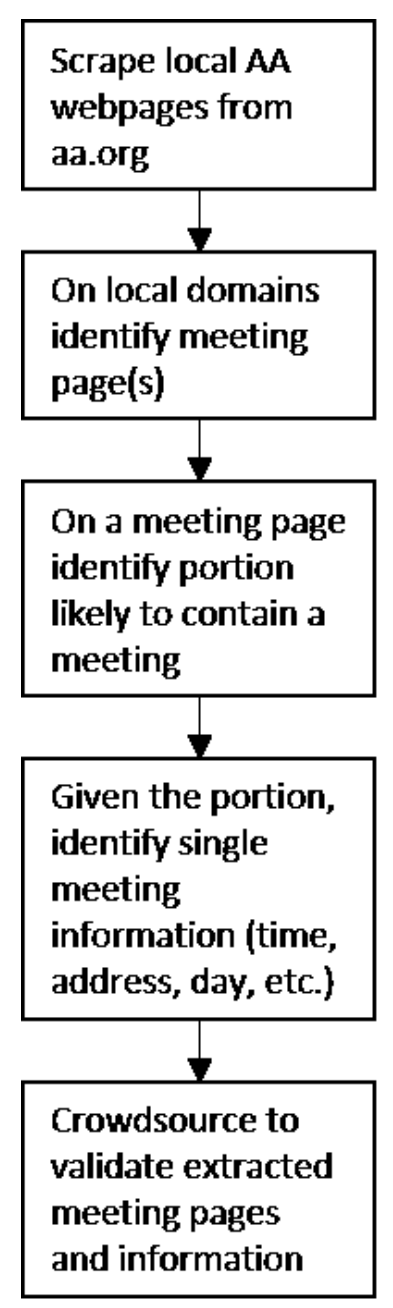

Figure 3: Flowchart of Developing a System to Create and Self-update of AA Meeting List meeting list. A web-scraping framework at first extracts information from different AA regional websites. The local websites have different pages describing the program, such as list of meetings and events, resources for newcomers etc. I train a binary classifier model to classify each of the pages to be either a meeting or a non-meeting page. This is necessary to avoid finding meeting information in many unrelated links. From the identified meeting pages, I search for repetitive patterns with time, day, and address information, as these are more likely to contain meeting information. As a next step, I am in the process of building a web interface where crowd-workers can contribute in updating the list continuously by:

- improving classification accuracy and recall

- validating if the extracted meeting information is right

- making correction if the day, time, or address information associated with a meeting is incorrect. A pipeline for this system is shown in Figure 3.

\section{Resources to Manage Information Overload for} Newcomers

Some of the participants revealed that getting a lot of new information just after starting to work the steps is sometimes an "information overload" for them. Besides, there are some variation in how the 12 traditions are followed in the meetings in different geographic location.

One way to help them overcome this problem is to design digital resources that support their endeavor in managing information overload. For example, a personal organizer mobile application can filter and prioritize useful information, as well as search and show context-based recommendation. Crowdsourcing can be utilized to find out geographic variation in the traditions.

List of Probable ITR Meeting Attendees

Online meeting attendees can interact with twelve-step members around the world, as ITR hosts over 100 different online meetings with attendees from more than 70 countries. Whereas some of the interview participants expressed their excitement to be exposed to people with diverse perspectives on recovery, others wanted to hear from likeminded others who follow similar practices. Besides, many of the meeting attendees want their online meetings to contain more people from their local meetings who they already knew. However, current online meetings on ITR do not offer a list of probable attendees making it difficult to choose a meeting based on personal preferences.

I designed a web browser extension that can work on the page of online meeting list on ITR. ITR users can confirm their attendances in a meeting beforehand as well as see the list of attendees (screenname and profile photo) that already confirmed their attendance. They can then access any of these profiles from the list to get an idea of the attendees' recovery history or other information. This would be useful for both newcomers and oldtimers to find meetings with particular set of attendees. Figure 4 shows the list of ITR online meetings after adding the extension.

\section{Conclusion}

Current communication technologies are ineffective to facilitate peer support in twelve-step groups. I identified some challenges faced by these groups and 


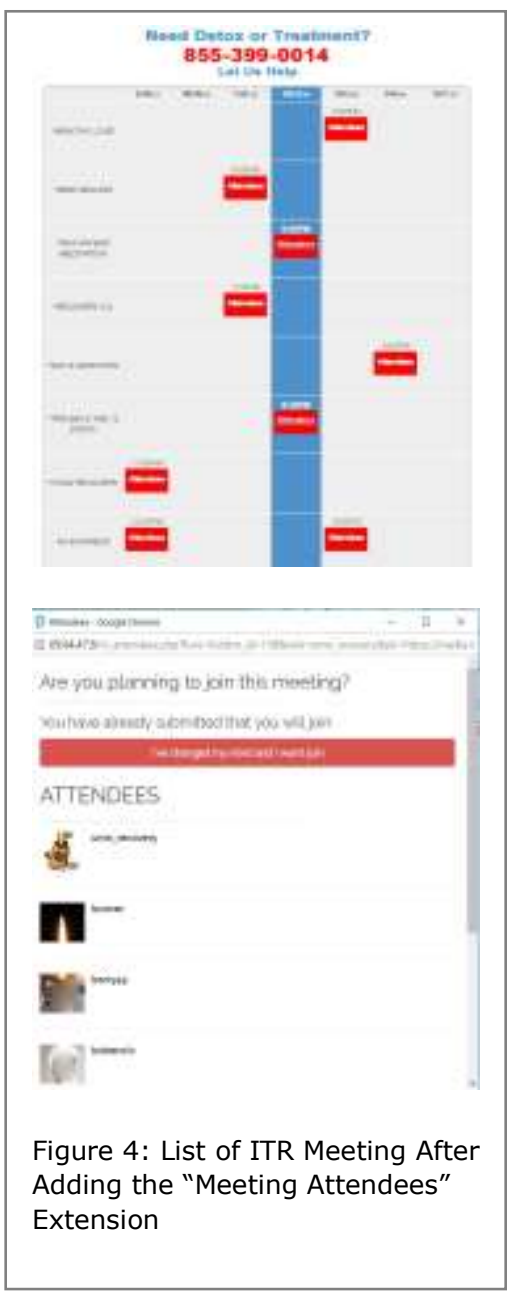

started working on designing technologies to support their need. The findings can help inform the design of technologies for peer-support in other health communities, too.

\section{Acknowledgements}

I would like to acknowledge my advisor, Svetlana Yarosh, for her support and feedback. Also, thanks to Baris Unver for his help with developing the extension. This work was funded by the NSF grant (1464376).

\section{References}

1. National Institute on Drug Abuse. 2015. Trends \& Statistics. Retrieved January 13, 2017 from https://www.drugabuse.gov/related-topics/trendsstatistics.

2. Lorraine R. Buis. 2008. Emotional and informational support messages in an online hospice support community. Computers, informatics, nursing: CIN 26 6: 358-367

3. Shelly Farnham, Lili Cheng, Linda Stone, et al. 2002. HutchWorld: Clinical Study of Computermediated Social Support for Cancer Patients and Their Caregivers. Proceedings of the SIGCHI Conference on Human Factors in Computing Systems, ACM, 375-382.

4. Keith Humphreys, Lee Ann Kaskutas, and Constance Weisner. 1998. The Alcoholics Anonymous Affiliation Scale: Development, Reliability, and Norms for Diverse Treated and Untreated Populations. Alcoholism: Clinical and Experimental Research 22, 5: 974-978.

5. Xiaoxiao Ma, Guanling Chen, and Juntao Xiao. 2010. Analysis of an Online Health Social Network.
Proceedings of the 1st ACM International Health Informatics Symposium, ACM, 297-306.

6. Diana MacLean, Sonal Gupta, Anna Lembke, Christopher Manning, and Jeffrey Heer. 2015. Forum77: An Analysis of an Online Health Forum Dedicated to Addiction Recovery. Proceedings of the 18th ACM Conference on Computer Supported Cooperative Work \& Social Computing, ACM, 15111526.

7. Sabirat Rubya and Svetlana Yarosh. 2017. VideoMediated Peer Support in an Online Community for Recovery from Substance Use Disorders. In Proc. of CSCW, To Appear.

8. Xi Wang, Kang Zhao, and Nick Street. 2014. Social Support and User Engagement in Online Health Communities. Smart Health, Springer International Publishing, 97-110.

9. Jennifer L. Welbourne, Anita L. Blanchard, and Marla D. Boughton. 2009. Supportive Communication, Sense of Virtual Community and Health Outcomes in Online Infertility Groups. Proceedings of the Fourth International Conference on Communities and Technologies, ACM, 31-40.

10.Svetlana Yarosh. 2013. Shifting Dynamics or Breaking Sacred Traditions?: The Role of Technology in Twelve-step Fellowships. Proceedings of the SIGCHI Conference on Human Factors in Computing Systems, ACM, 3413-3422. 